\title{
A-scan ultrasound system for real-time puncture safety assessment during percutaneous nephrolithotomy
}

\author{
Pedro L. Rodrigues*a,b,c ${ }^{\text {a }}$ Nuno F. Rodrigues ${ }^{\mathrm{b}, \mathrm{c}}$, Jaime C. Fonseca ${ }^{\mathrm{b}}$, M. A. von Krüger ${ }^{\mathrm{d}}$, W. C. A. \\ Pereira $^{\mathrm{d}}$, João L. Vilaça ${ }^{\mathrm{a}, \mathrm{c}}$ \\ ${ }^{\mathrm{a}}$ ICVS/3B's - PT Government Associate Laboratory, Braga/Guimarães, Portugal; ${ }^{\mathrm{b}}$ Algoritmi Center, \\ School of Engineering, University of Minho, Guimarães, Portugal; ${ }^{c}$ DIGARC - Polytechnic Institute \\ of Cávado and Ave, Barcelos, Portugal; ${ }^{\mathrm{d} B i o m e d i c a l ~ E n g i n e e r i n g ~ P r o g r a m ~-~ C O P P E / U F R J, ~ R i o ~ d e ~}$ \\ Janeiro, Brasil
}

\begin{abstract}
Background: Kidney stone is a major universal health problem, affecting $10 \%$ of the population worldwide. Percutaneous nephrolithotomy is a first-line and established procedure for disintegration and removal of renal stones. Its surgical success depends on the precise needle puncture of renal calyces, which remains the most challenging task for surgeons. This work describes and tests a new ultrasound based system to alert the surgeon when undesirable anatomical structures are in between the puncture path defined through a tracked needle.
\end{abstract}

Methods: Two circular ultrasound transducers were built with a single 3.3-MHz piezoelectric ceramic PZT SN8, 25.4 $\mathrm{mm}$ of radius and resin-epoxy matching and backing layers. One matching layer was designed with a concave curvature to work as an acoustic lens with long focusing. The A-scan signals were filtered and processed to automatically detect reflected echoes.

Results: The transducers were mapped in water tank and tested in a study involving 45 phantoms. Each phantom mimics different needle insertion trajectories with a percutaneous path length between 80 and $150 \mathrm{~mm}$. Results showed that the beam cross-sectional area oscillates around the ceramics radius and it was possible to automatically detect echo signals in phantoms with length higher than $80 \mathrm{~mm}$.

Conclusions: This new solution may alert the surgeon about anatomical tissues changes during needle insertion, which may decrease the need of X-Ray radiation exposure and ultrasound image evaluation during percutaneous puncture.

Keywords: guided surgery, percutaneous puncture, motion tracking sensors, ultrasound transducer, renal access, percutaneous nephrolithotomy

\section{INTRODUCTION}

Nephrolithiasis is a major universal health problem that affects $10 \%$ of the population worldwide with a recurrence rate close to 50\% [1-3]. Percutaneous nephrolithotomy (PNL) is a first-line and established surgical procedure, even in cases of ectopic or malformed kidneys, regardless of calculi location, size and number [1,4].

PNL puncture stage still remains the most challenging task for surgeons, mainly due to the anatomic target restricted vision, the difficulty on handling surgical instruments, the restrictive mobility inside the human body, and constant anatomic target, tissue and organs deformations and movements [5, 6]. An inaccurate needle puncture can lead to serious complications such as injuries in the kidney and contiguous organs and, eventually, jeopardize the overall surgical procedure and treatment outcome.

New approaches have been emerging in the urology domain regarding the current trend towards minimally invasive surgery and in an attempt to overcome the PNL weaknesses mentioned above [7]. In order to continuously track the needle tip inside the human body, imageless techniques are never used at PNL. Real-time images from computed tomography (CT) and fluoroscopy $\mathrm{C}$-arm lead to a significant increase in radiation exposure. On its turn, ultrasounds (US) and magnetic resonance (MR) have proven to be an advantageous imaging option by providing radiation-free realtime imaging. However, their targeting ability for small calculi is limited [7, 8]. Moreover, MR is also expensiveness, needs large spaces and compatible surgical instruments [9]. Finally, most of US and C-arm systems, only produce 2D images not providing information about the $3 \mathrm{D}$ target position and surrounding tissues $[7,10,11]$. 
Often, multiple images from different sources are combined in a single view to increase the manipulated anatomic structures information $[12,13]$. Although these multimodal images can improve PNL, they need a pre-processing step to register all the anatomic structures through non-rigid or rigid algorithms. Only the non-rigid algorithms support the alignment of tissues and organs taking into account different deformation degrees. However, these algorithms are still an active research area, lacking on robustness, precision and real-time methods [12].

Concerning the literature pitfalls, one proposes a solution based in a new US probe specifically develop for this work. By automatically comparing A-scanned signals with prior references, this system will alert the surgeon when unwanted anatomical structures are in between the puncture path. This new probe was tested in US phantom blocks which mimics different situations during percutaneous renal access.

\section{METHODS}

\subsection{System overview}

The proposed framework is based in electromagnetic tracking sensors (EMT) coupled to surgical instruments and an ultrasound probe specifically developed for this work. As already described by the authors in [14], firstly, a catheter will be placed, through an ureterorenoscope with a camera and guided tip, into the puncture target. This catheter will have a position and orientation electromagnetic sensor on its tip that will function as a real-time target locator. Then, a needle that integrates a similar sensor will be used for the puncture procedure. From the data provided by both sensors, it is possible to define the percutaneous puncture trajectory (PPT) from where the needle will be inserted. Although this methodology is able to easily guide the surgeon along a percutaneous path, it cannot assure if any anatomical structure stands in between the PPT.

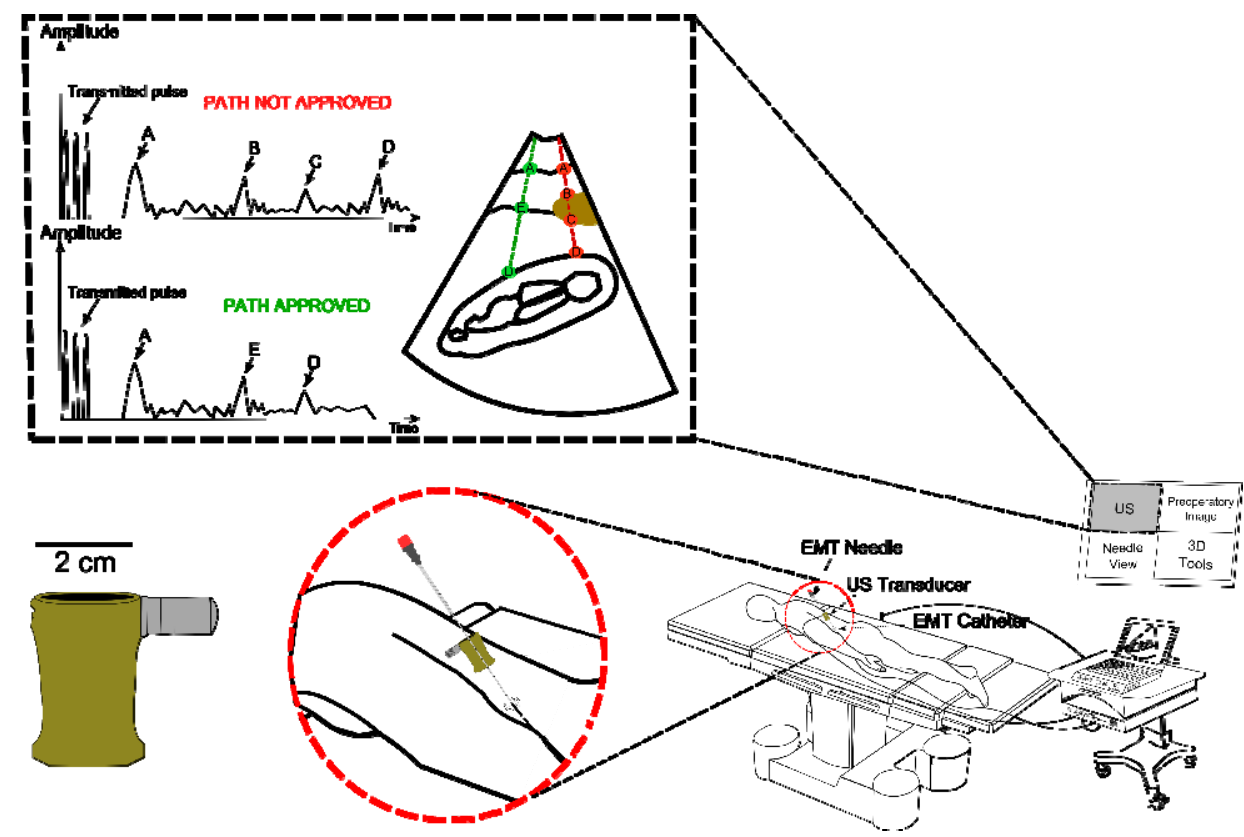

Figure 1. Surgical setup and representation of different reflected pulses from tissue boundaries showing an adequate or inadequate puncture path.

In this work, one is improving the previous framework using A-scanned signals received from a new US probe. Considering the generated PPT, the surgeon must evaluate if any vital organ is in between the percutaneous path that might compromise the surgical success using an imaging system such as ultrasound or fluoroscopy C-arm. After this image checking, the presented probe should be aligned with the puncture needle. To this extent, the probe was designed with small dimension, so that it can be easily oriented with the puncture needle (Figure 1).

From this point, the surgeon must follow the PPT shown in a 3D software, where the echoes from the presented probe will be processed in order to alert the surgeon whether tissue interfaces changes during puncture. 


\subsection{Phantom Building}

Although PNL puncture has proven to be very safe with medical imaging, as in any surgical procedure there are risks and potential complications. Spleen, liver, lung, colon and pancreas are the most common organs to be injured. $45 \mathrm{n}$ layer phantoms were manufactured based on a study of 65 computed tomography images across puncture trajectories (Fig. 1-a). Different thicknesses between skin puncture site and kidney target were calculated each time the puncture path intersected a new organ (Points P1 to P10 and PT in Fig. 1) from the skin points (P1 and P7) towards a kidney target (PT). The mean path distance was $123.8 \pm 25.2 \mathrm{~mm}$ (from the skin puncture site to the kidney interface, P1 to P6 and P7 to P10) and $154.5 \pm 26.2 \mathrm{~mm}$ (from the skin puncture site to the kidney calculi, P1 to PT and P7 to PT). Commonly, the organs along the puncture path are intersected with thicknesses ranging from 5 to $30 \mathrm{~mm}$.

After this study, different phantom blocks were selected and stacked together according to the acoustical tissue properties found in the literature [15-17]. All phantoms were fabricated with PVCP mixed with different concentrations of graphite powder $(0.3 \%, 0.5 \%, 0.7 \%$ and $1 \%)$. The mixture was put in a vacuum chamber for about 40 minutes, where it was stirred to release trapped air bubbles. Finally, the mixture was heated until it reached $180^{\circ}$ and then stabilized in room temperature (Fig. 1-b)).

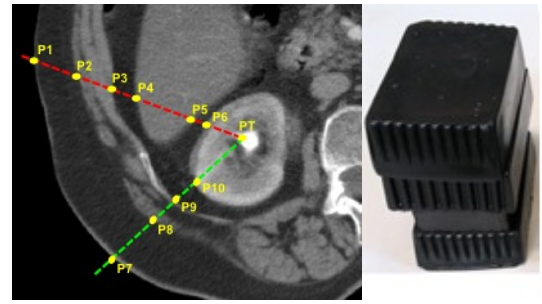

a)

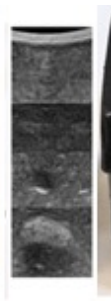

b)

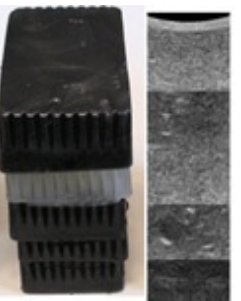

Figure 2. a) Representation of two different puncture trajectories: puncture path where the liver is perforated and an ideal puncture path; b) testing phantom blocks.

\subsection{US Transducer - Fabrication}

Two ultrasonic transducers were designed and fabricated to generate enough acoustical power to detect tissue interfaces along the percutaneous path. To fabricate such transducers, one choose a PZT SN8 ceramic disc with nominal frequency of $3.3 \mathrm{MHz}, 25.4 \mathrm{~mm}$ of radius and $7 \mathrm{~mm}$ of thickness, which assures enough power to achieve the desired length of approximately $120 \mathrm{~mm}$. The fabrication process started with the design and manufacture of a cylindrical mold to hold the ceramic, backing and matching ultrasonic layers. The transducers were fabricated as follows:

a) For each ceramic, electrical contacts were connected between front and back ceramic faces (ground and excitation, respectively) and a $\mathrm{BNC}$ connector;

b) For each transducer, the ceramic and contacts were coupled to the mold and placed in an aluminum metal tube (outer diameter of $32 \mathrm{~mm}$ ) for supporting;

c) The matching layers were made with epoxy resin mixed with tungsten powder (1 micron and $99.95 \%$ purity), and put in a vacuum chamber for 40 minutes to release trapped air bubbles. These layers were designed and fabricated to have a thickness of $\lambda / 4$ (where $\lambda$ is the US wavelength). This kind of matching layers have high efficiency response with Gaussian shaped pass-bands [18, 19];

d) The matching layers were cured at room temperature during two days. They were cured in designed molds allowing a layer thickness for the wanted resonance frequency. One mold had a convex curvature allowing the formation of a concave matching layer that worked as a low focusing lens;

e) The backing layers were made with epoxy resin mixed with tungsten and cork powders. These mixtures were not put in the vacuum chamber because one aimed at high acoustic attenuation so as to eliminate the back-wall reflections (layer also stabilized at room temperature during two days);

f) Assessment of the electrical impedance curves (magnitude and phase) as a function of frequency using an impedance analyzer Omicron Lab Bode 100;

g) Design of an impedance matching electrical circuit to improve and maximize the power of the acquired signal. 


\subsection{US Transducer - Performance Mapping}

Both US transducers were mapped in an acoustic tank with a needle hydrophone (Precision Acoustics Ltd.) with a diameter of $0.5 \mathrm{~mm}$ and 200.7 sensitivity $\mathrm{mV} / \mathrm{MPa}$. The transducer was excited by 5-cycle bursts of $3.38 \mathrm{MHz}$, amplitude $10 \mathrm{Vpp}$ by a generator AFG 3021 from Tektronix.

The hydrophone was moved inside the water tank in a 50x50x150 mm volume by a XYZ robotic table with a step of $0.348,0.348$ and $25 \mathrm{~mm}$ in $\mathrm{Ox}$, Oy and $\mathrm{Oz}$, respectively. The received signal was digitalized and stored in a $\mathrm{PC}$ in Labview ${ }^{\circledR}$ (National Instruments) that controls an oscilloscope (TDS 3014B Tektronix).

The signals were averaged 8 times with a sampling frequency of $80 \mathrm{MHz}$ and 15.000 points/signal. This mapping allows to evaluate the focal length (distance from the transducer face to the focus) and beam cross-sectional area (area on the surface of a plane perpendicular to the beam axis consisting of all points where the pulse intensity integral is greater than 25 percent of the maximum pulse intensity integral).

\subsection{US Transducer - Echo Detection}

These US transducers were tested in US phantom (Fig. 2) which signals were acquired using the US-Key® equipment. In order to automatically generate an alert every time that some new anatomical interface appear in between the PPT the following methodology was used:

a. At the beginning of the puncture procedure, the US probe is oriented with the puncture needle;

b. The US probe starts acquiring signals;

c. An A-scan signal, $S_{\text {init }}$, is acquired at the beginning of the puncture step. Considering that at this stage, any vital organ is in between the puncture path, $\mathrm{S}_{\text {init }}$ will be used for further comparisons;

d. During needle insertion, new A-scan signals, $S_{\text {new }}$, are acquired and compared with $S_{\text {init. }}$

All A-signals were acquired with 15 frames per second (fps). The median and average of 8 frames were used to eliminate random and unwanted interface echoes. The outcome signal was further filtered using a low-pass Butterworth filter (cut frequency of $3.1 \mathrm{MHz}$ ). The peak position, corresponding to an anatomical interface, were calculated where the average signal curvature over an echo duration (2us) region was concave down.

After the filtering process, an echo create an alert if it was not present in the starting $S_{\text {init }}$ signal and if it remains detected in current signal $\mathrm{S}_{\text {new }}$ more than a certain period of time. During experimental tests, this time was empirically calculated as being 2 seconds.

\section{RESULTS}

Fig. 3 a) and b) shows the US beam amplitude map at $100 \mathrm{~mm}$ away from the transducer surface. The beam crosssectional area oscillates around the ceramic area $\left(25 \mathrm{~mm}^{2}\right)$ for the transducer without lens.

On the other hand, the beam for the transducer with lens was narrowed to $70 \%$ of the ceramic area. Fig. 3 c) and d) shows the measured electrical impedance as a function of frequency for the ceramic element in air. After applying an impedance matching electrical circuit both transducers showed a phase near $0^{\circ}$ and a magnitude about $50 \Omega$ at the resonance frequency of $3.38 \mathrm{MHz}$. The beam power of the transducer with lens was increased $80 \%$ with the impedance matching circuit.

Experimental tests also showed that beam amplitude was reduced more than $50 \%$ when the interface angle was higher than $18^{\circ}$ for both transducers. 


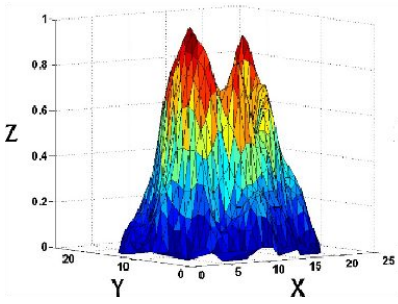

a)

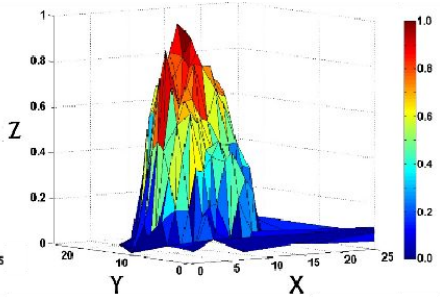

b)

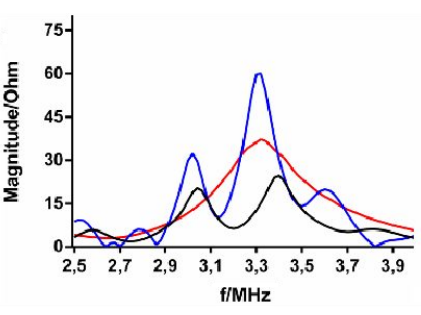

c)

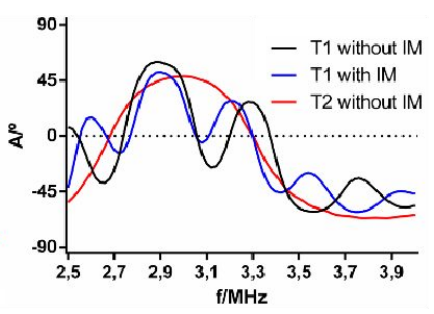

d)

Figure 3. a) and b) show the US beam mapping (distance=75 mm) without and with lens, respectively; c) and d) show the electrical magnitude and phase, respectively, for both transducers measured in air $(\mathrm{T} 1=$ transducer without lens, $\mathrm{T} 2=$ transducer with lens and $\mathrm{IM}=$ impedance matching circuit.

Pulse-echo measurements were performed in different phantom setups (Fig. 2). When comparing to the ground truth signals, the transducer without lens (Fig. 4-a) was able to detect echoes up to $120 \mathrm{~mm}$ and showed an average sensitivity of $67.5 \% \pm 7.3 \%$. On the other hand, the transducer with lens (Fig. 4-c) was only able to detect echoes up to $80 \mathrm{~mm}$, but with an average sensitivity of $73.2 \%+\% \pm 6.4 \%$.

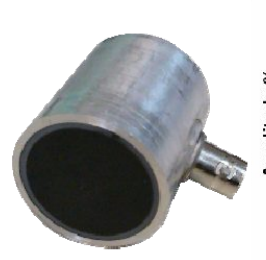

a)

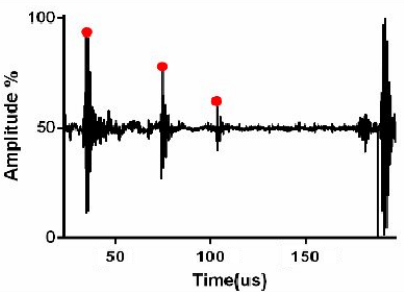

b)

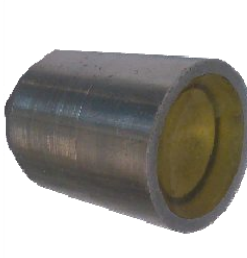

c)

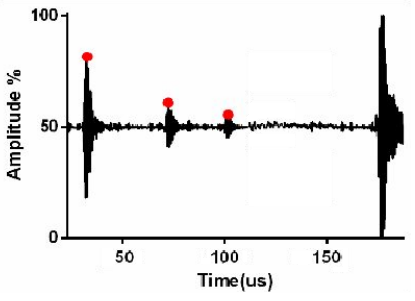

d)

Figure 4. a) Transducer without lens and its echo response in b); c) transducer with lens and its echo response in d).

\section{DISCUSSION}

Inaccurate punctures can cause damage in kidneys and its surrounding tissues and organs [4, 7]. The learning curve for PCNL is estimated to be around 60 cases, a situation that makes complication rates increase during the long surgeons training period [20]. The needle trajectory in puncture processes cannot be changed once the needle is inserted to a depth

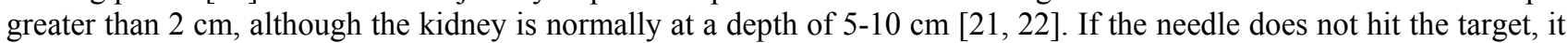
has to be removed from the human body and reinserted again. So, one may need to make several attempts to accomplish a correct needle insertion, consequently causing an increase in postoperative complications, poorer surgical outcomes and increased likelihood of surrounding structures perforation.

The proposed methodology makes use of a catheter and needle, both integrating an Aurora EMT sensor at its tip, to retrieve in real-time its position and orientation. The catheter remained associated to the puncture target (worked as a 3D real-time locator) and was permanently monitored by the EMT sensor. Therefore it followed in real-time all the deformations and movements.

Since ultrasound is a versatile technology, low-cost, radiation-free and allows real-time signals, the presented work focuses in a new US probe to alert if the PPT changes during needle insertion. The piezoelectric ceramic PZT SN8 with 25.4-mm radius was adequately to create an ultrasound beam that produces detectable echoes in different US phantom interfaces.

In contrast with clinical current practice, the needle can be inserted from a predefined and safe path. On the other hand, it may reduce the need to acquire or interpret new images during needle insertion, e.g. by ultrasound or C-arm, to evaluate if any organ is positioned in between the PPT as a result of tissue and organs movements or deformations. This may lead to a reduction in the puncture time or decrease in the radiation exposure for both the patient and surgeon. 
However, in vivo testing is necessary as future work. Another research path may include an optimization of the ultrasound matching and backing layers in order to reduce the level of ripples in the spectra and increase the efficiency of the methodology.

\section{CONCLUSION}

Due to the high number of patients suffering from nephrolithiasis and the predictable growth of PNL practice, any system that may improve surgical outcomes is a potentially attractive solution. This work presents an ultrasound that might improve percutaneous puncture by alerting the surgeon when the interfaces along the puncture path changes. It is expected that such a system may reduce X-Ray radiation, time for puncture procedure and increase its safety.

\section{ACKNOWLEDGEMENT}

The authors acknowledge to Foundation for Science and Technology (FCT) - Portugal for the fellowships with references: SFRH/BD/74276/2010 and the Brazilian agencies CAPES and CNPq. The present submission corresponds to original research work of the authors and has never been submitted elsewhere for publication.

\section{REFERENCES}

[1] C. M. Cracco, C. M. Scoffone, and R. M. Scarpa, "New developments in percutaneous techniques for simple and complex branched renal stones," Curr Opin Urol, (2011).

[2] A. Skolarikos, G. Alivizatos, and J. J. M. C. H. de la Rosette, "Percutaneous nephrolithotomy and its legacy," European Urology, 47(1), 22-28 (2005).

[3] E. M. Worcester, and F. L. Coe, "Calcium Kidney Stones," New England Journal of Medicine, 363(10), 954963 (2010).

[4] M. S. Michel, L. Trojan, and J. J. Rassweiler, "Complications in percutaneous nephrolithotomy," European Urology, 51(4), 899-906 (2007).

[5] H. R. H. Patel, A. Linares, and J. V. Joseph, "Robotic and laparoscopic surgery: Cost and training," Surgical Oncology-Oxford, 18(3), 242-246 (2009).

[6] P. Gomes, "Surgical robotics: Reviewing the past, analysing the present, imagining the future," Robotics and Computer-Integrated Manufacturing, In Press, Corrected Proof.

[7] C. Kalogeropoulou, P. Kallidonis, and E. N. Liatsikos, "Imaging in Percutaneous Nephrolithotomy," Journal of Endourology, 23(10), 1571-1577 (2009).

[8] H. Karami, A. H. Arbab, A. Rezaei et al., "Percutaneous nephrolithotomy with ultrasonography-guided renal access in the lateral decubitus flank position," Journal of Endourology, 23(1), 33-5 (2009).

[9] D. P. Perrin, N. V. Vasilyev, P. Novotny et al., "Image Guided Surgical Interventions," Current Problems in Surgery, 46(9), 730-766 (2009).

[10] K. R. Ghani, U. Patel, and K. Anson, "Computed tomography for percutaneous renal access," Journal of Endourology, 23(10), 1633-9 (2009).

[11] K. Cleary, and T. M. Peters, "Image-guided interventions: technology review and clinical applications," Annu Rev Biomed Eng, 12, 119-42 (2010).

[12] P. Markelj, D. Tomazevic, B. Likar et al., "A review of 3D/2D registration methods for image-guided interventions," Med Image Anal, (2010).

[13] G. P. Penney, J. M. Blackall, M. S. Hamady et al., "Registration of freehand 3D ultrasound and magnetic resonance liver images," Medical Image Analysis, 8(1), 81-91 (2004).

[14] P. L. Rodrigues, J. L. Vilaça, C. Oliveira et al., "Collecting system percutaneous access using real-time tracking sensors: First pig model in vivo experience,” The Journal of urology, 190(5), 1932-1937 (2013). 
[15] F. A. Duck, [Physical properties of tissues: a comprehensive reference book] Academic Press, (1990).

[16] B. A. Angelsen, [Ultrasound imaging: waves, signals, and signal processing. 1. Basic principles wave generation, propagation, and beamforming in homogeneous tissue] Emantec, (2000).

[17] K. Zell, J. Sperl, M. Vogel et al., "Acoustical properties of selected tissue phantom materials for ultrasound imaging," Physics in medicine and biology, 52(20), N475 (2007).

[18] C. S. Desilets, J. D. Fraser, and G. S. Kino, "The design of efficient broad-band piezoelectric transducers," Sonics and Ultrasonics, IEEE Transactions on, 25(3), 115-125 (1978).

[19] A. Bybi, C. Granger, S. Grondel et al., "Electrical method for crosstalk cancellation in transducer arrays," NDT \& E International, 62(0), 115-121 (2014).

[20] J. J. M. C. H. de la Rosette, M. P. Laguna, J. J. Rassweiler et al., "Training in Percutaneous Nephrolithotomy-A Critical Review,” European Urology, 54(5), 994-1003 (2008).

[21] P. Mozer, P. Conort, A. Leroy et al., "Aid to percutaneous renal access by virtual projection of the ultrasound puncture tract onto fluoroscopic images," Journal of Endourology, 21(5), 460-465 (2007).

[22] J. Lazarus, and J. Williams, "The Locator: novel percutaneous nephrolithotomy apparatus to aid collecting system puncture--a preliminary report," Journal of Endourology, 25(5), 747-50 (2011). 